\title{
The ROSAT deep survey
}

\section{RX J105343+5735: A massive cluster at $z=1.263$}

\author{
D. Thompson ${ }^{1}$, L. Pozzetti ${ }^{2}$, G. Hasinger ${ }^{3}$, I. Lehmann ${ }^{3,4}$, M. Schmidt ${ }^{1}$, B. T. Soifer ${ }^{1,5}$, G. Szokoly ${ }^{3}$, \\ G. Wilson ${ }^{6}$, and G. Zamorani ${ }^{2}$ \\ 1 Palomar Observatory, California Institute of Technology, MS 320-47 Pasadena, CA 91125, USA \\ 2 Osservatorio Astronomico di Bologna, Via Ranzani 1, 40127 Bologna, Italy \\ 3 Astrophysikalisches Institut Potsdam, An der Sternwarte 16, 14482 Postdam, Germany \\ 4 Department of Astronomy \& Astrophysics, 525 Davey Laboratory, The Pennsylvania State University, \\ University Park, PA 16802, USA \\ 5 SIRTF Science Center, California Institute of Technology, Pasadena, CA 91125, USA \\ 6 Physics Department, Brown University 182 Hope Street Providence, RI 02912, USA
}

Received 14 May 2001 / Accepted 16 July 2001

\begin{abstract}
The eastern lobe of the unusual double-lobed extended X-ray source RX J105343+5735 is confirmed to be a massive cluster at high redshift. Deep optical and near-infrared imaging show an overdensity of galaxies in both X-ray lobes, including a significant excess of red galaxies $(R-K>5.3)$ with colors typical of elliptical galaxies at $z>1$. We have used new photometry to place better constraints on the redshifts of the cluster galaxies. A Keck NIRSPEC spectrum of one of the bright central galaxies in the eastern lobe shows a narrow $\mathrm{H} \alpha$ emission line at 1.485 microns, yielding a redshift of 1.263 . The [O II] $\lambda 3727 \AA$ line from the gravitationally lensed arc is also detected, giving a redshift of 2.577 for the lensed galaxy and confirming prior measurements. The improbability of chance alignment and similarity of colors for the galaxies in the two X-ray lobes are consistent with the western lobe also being at $z=1.26$. The system may thus represent a pair of clusters in the process of merging.
\end{abstract}

Key words. galaxies: clusters, distances and redshifts - cosmology: observations, gravitational lensing infrared: galaxies - X-rays: galaxies

\section{Introduction}

Galaxy clusters are the most massive bound structures in the Universe. The timing of cluster formation provides strong constraints on the fundamental cosmological parameters, thereby driving the search for clusters at high redshift $(z>1)$. Clusters are the second most abundant class of X-ray sources, after active nuclei, detected through their hot X-ray emitting intracluster gas. They are visible in deep X-ray surveys out to high redshifts. Several clusters are known at $z>1$ which have been either selected from X-ray surveys (Rosati et al. 1999, 2000) or subsequently detected in X-rays after identification at longer wavelengths (Stanford et al. 1997; Benítez et al. 1999). There is also a tentative X-ray detection of extended cluster gas around a $z=2.156$ radio galaxy by Carilli et al. (1998).

In this paper we report on additional observations of the faint, double-lobed X-ray source RX J105343+5735

Send offprint requests to: D. Thompson, e-mail: djt@mop.caltech.edu
(Hasinger et al. 1998, hereafter Paper IV), detected in the ROSAT Ultra Deep Survey of the Lockman Hole (Lehmann et al. 2001). We assume an Einsteinde Sitter cosmology, with $\Omega_{M}=1, \Omega_{\Lambda}=0$; with $H_{0}=50 \mathrm{~km} \mathrm{~s}^{-1} \mathrm{Mpc}^{-1}$, unless otherwise noted.

\section{Observations}

\subsection{Imaging}

In addition to the Keck $R$-band data presented in Paper IV, $V$ and $I$ band images were obtained on UT 1997 April 4-5 with the University of Hawaii's UH8K Mosaic Camera at the prime focus of the CFH $3.6 \mathrm{~m}$ telescope. In this configuration, the camera has a field size of 0.5 square; only a small subset was used for this project. The $I$-band image of the RX J105343+5735 field is shown in Fig. 1, overlaid with the ROSAT HRI X-ray contours and the outlines of the areas covered by the infrared data (see below). A total of two hours in each band was taken, comprised of several shorter exposures dithered on the sky. A description of the reduction of these data can be found in 
Wilson \& Kaiser (in prep.). The data were calibrated onto the Vega scale using standard stars from Landolt (1992). All of the optical data have been corrected for distortions and resampled to 0 "' 15 per pixel to correspond to the infrared images.

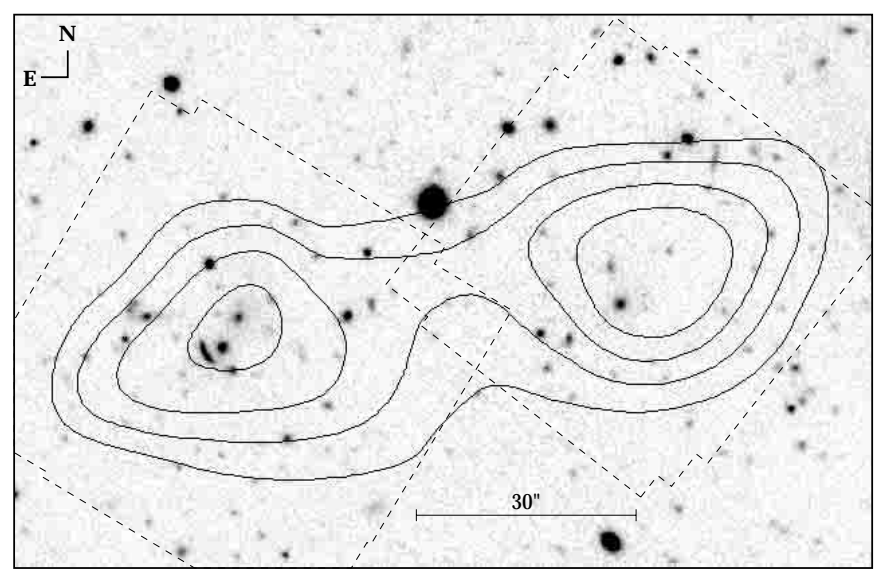

Fig. 1. An $I$-band image of the RX J105343+5735 field, overlaid with the ROSAT HRI Image X-ray contours (solid lines, with contours at $3,4,5$, and $6 \sigma$ ) and the areas covered by the near-infrared imaging (dashed lines).

Deep near-infrared images of RX J105343+5735 were obtained with the facility Near-Infrared Imaging Camera (NIRC, Matthews \& Soifer 1994) on the Keck I telescope. NIRC uses a $256^{2} \mathrm{InSb}$ detector at 0 "' 15 per pixel, for a $38^{\prime \prime} .4$ square field of view. The western lobe was observed on UT 1999 March 30 in the JHK bands in non-photometric weather. The eastern lobe was observed on UT 1999 June 4 in the $z J H K$ bands under photometric conditions and 0.5 arcsec seeing. Images were sky-subtracted and flatfielded, then stacked using integer pixel offsets. The data were calibrated onto the Vega scale using the Persson et al. (1998) infrared standard stars. The $5 \sigma K$-band point source detection limits in two arcsecond diameter apertures are $K=21.12$ (east) and $K=20.24$ (west). The central $30^{\prime \prime}$ square from the $K$-band images of each X-ray lobe are shown in Fig. 2.

Aperture photometry in the VRIzJHK bands was extracted for all of the galaxies visible in the two Keck $K$ band images. We used a two arcsec diameter aperture to minimize contamination from nearby objects. For the lensed source we used an aperture approximating the curved shape of the arc (about $1^{\prime \prime} .2$ by 3 ". 6 ). No corrections were made for the seeing, which ranges from 0.75 in the $V R I$ data to 0.5 in the near-infrared. The $K$-band data are complete for $K<21$ in the eastern lobe and $K<20$ in the western lobe.

\subsection{Spectroscopy}

A near-infrared spectrum of the brightest galaxy in the eastern lobe of RX J105343+5735, denoted object \#4 in Paper IV, was obtained on UT 2000 March 20 with the near infrared spectrograph NIRSPEC (McLean et al. 1998) on the Keck II telescope. We used a $0 !$ '76 (4 pixel) slit. Due to technical problems, only a single $600 \mathrm{~s}$ exposure was obtained on-source; a second $600 \mathrm{~s}$ exposure was obtained off-source for sky subtraction. Atmospheric $\mathrm{OH}$ emission lines were used for the wavelength calibration. A short exposure of the G6V star $\mathrm{BD}+44^{\circ} 2155$ at similar airmass was used to correct for atmospheric absorption. The slit, oriented east-west, also covered the northern end of the giant arc.

\section{Discussion}

\subsection{Galaxy overdensities}

From average galaxy counts for field galaxies in the $K$ band (Hall et al. 1998) we estimate an overdensity of a factor of about 5 in the two regions of $40^{\prime \prime} \times 40^{\prime \prime}$ centered on the eastern and western lobes of RX J105343+5735.

However, most of these galaxies have fairly red optical to near-infrared colors, typical of passively evolving ellipticals at $z>1$. They show a clear color-magnitude sequence typical of cluster ellipticals (Fig. 3), similar in the two lobes and to those detected in confirmed high-redshift clusters at $z=1.26$ (Rosati et al. 1999) and at $z=1.27$ (Stanford et al. 1997).

Daddi et al. (2000) has determined the surface density of red galaxies in the field, most of which are likely to be high-redshift elliptical galaxies. Taking similar cuts in color $(R-K>5.3)$ and magnitude $(K<19.2)$ in both samples for comparison, we see a significant overdensity of red galaxies: we find 14 galaxies in our field, while only 0.35 are expected from the field sample in the same area. This strongly suggests the presence of a highredshift cluster of galaxies in our field.

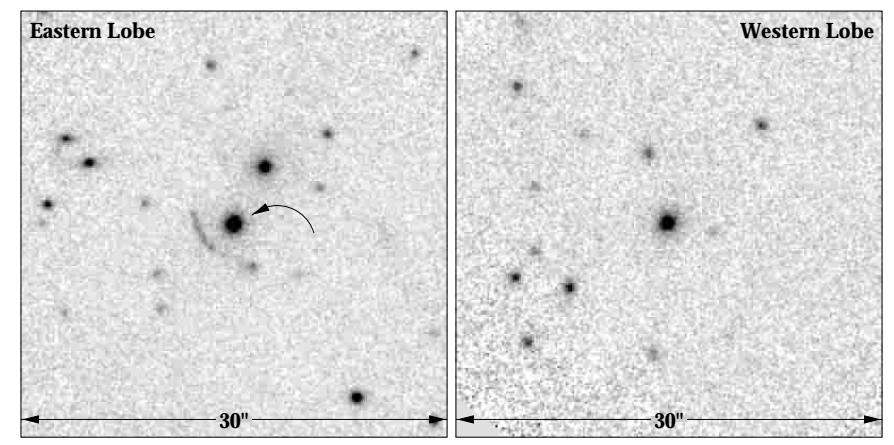

Fig. 2. $K$-band images centered on the brightest galaxy in each of the two X-ray lobes of RX J105343+5735. The galaxy in the Eastern lobe for which we obtained a spectroscopic redshift is identified with the arrow.

\subsection{Photometric redshifts}

To estimate the redshifts of faint galaxies we used a standard photometric redshift technique. In our revised version of the software, the templates consist of a set of 


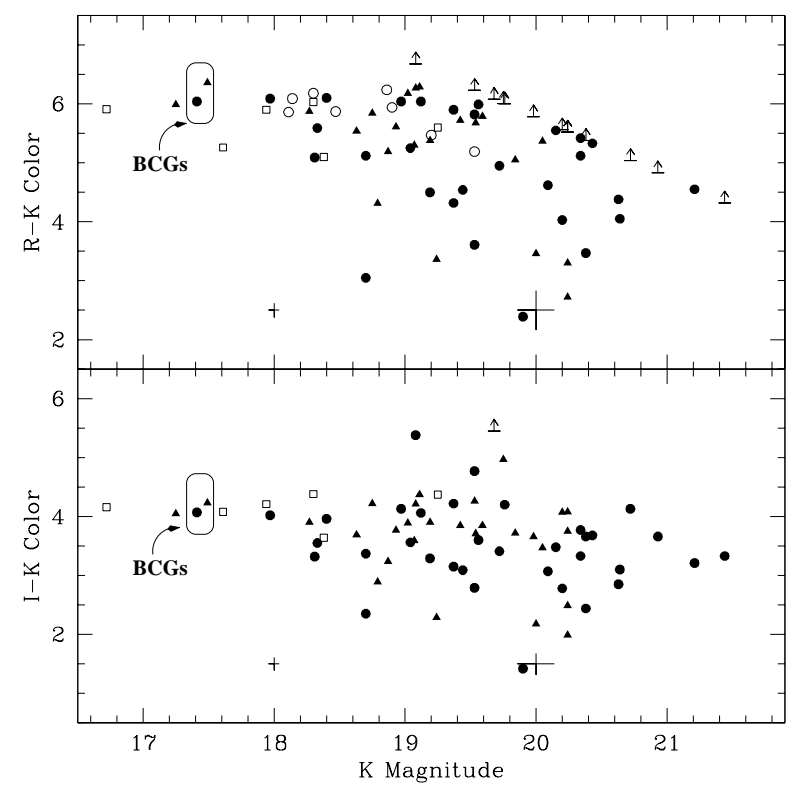

Fig. 3. Color-magnitude diagrams for the RX J105343+5735 field, showing a strong red color sequence. Data from the eastern lobe are plotted with filled circles, the western lobe with filled triangles. The brightest cluster galaxies are marked, and the average $1 \sigma$ uncertainties in the $K$-band photometry for the eastern lobe are indicated for $K=18$ and 20. Objects undetected in $R$ (arrows) are plotted assuming $R=25.76$, the $5 \sigma$ limit of our $R$-band data. For comparison, we also plot data from two other confirmed clusters at similar redshift: Rosati et al. (1999), open squares, and Stanford et al. (1997), open circles.

synthetic spectra from GISSEL 1998 (Bruzual \& Charlot 1993) with four different star formation histories: a deltafunction burst (simple stellar population - SSP), a constant star formation rate (SFR), and 2 exponentially decreasing SFRs $(\tau=0.3,1$ Gyrs $)$. The models span a wide range of ages, with 32 steps in age from $10^{5}$ to $2 \times 10^{10} \mathrm{yr}$. The basic set of templates includes only solar metallicity and Salpeter's IMF $\left(x=1.35,0.1<m<125 M_{\odot}\right)$. We include the effects of attenuation by the intergalactic medium (Madau 1995), which is extremely important at high redshift, along with internal dust absorption. We adopt a dust-screen model and the small Magellanic cloud extinction law with $E(B-V)$ ranging from 0.0 to 0.5 in steps of 0.1 .

The photometric redshift $\left(z_{\mathrm{ph}}\right)$ for each galaxy was computed by applying a standard, error-weighted $\chi^{2}$ minimization procedure. Upper limits are set at the $5 \sigma$ level for objects not detected in a given band. The uncertainties on $z_{\mathrm{ph}}$ corresponding to the $68 \%$ and $90 \%$ confidence levels are computed by means of the $\Delta \chi^{2}$ increment for a single parameter (Avni 1976).

The observed broad-band SED of a given galaxy is compared to our set of template spectra as:

$$
\chi^{2}(z)=\sum_{i=1}^{N_{\text {filters }}}\left[\frac{m_{\mathrm{obs}, i}-m_{\mathrm{temp}, i}(z)-a(z)}{\sigma_{i}}\right]^{2}
$$

where $m_{\text {temp }, i}$ is the template magnitude in filter $i$, and $m_{\mathrm{obs}, i}$ and $\sigma_{i}$ are the observed magnitude and uncertainty in filter $i$. A normalization constant is incorporated in the factor $a$.

We tested the software using about 100 galaxies in the HDF-N from Fernandez-Soto et al. (1999), with $I_{814}<$ 25 and known spectroscopic redshifts from Steidel et al. (1996) and Lowenthal et al. (1997). Photometry for these galaxies is available in the $U_{300}, B_{450}, V_{606}$, and $I_{814}$ bands from HST images (Williams et al. 1996) and in the $J, H$, and $K$ bands from Kitt Peak data (Connolly et al. 1997). These tests indicate that, at low redshift $(z<1.5)$, there is a relatively low dispersion in redshift $\left(\delta_{z}=\sigma_{z} /(1+\right.$ $\langle z\rangle)=0.07)$ as well as a low fraction of catastrophic errors $(c=2 \%)$, defined as those having $\left|z_{\mathrm{sp}}-z_{\mathrm{ph}}\right|>3 \sigma_{z}$. For higher redshifts $(z>1.5)$ these numbers are slightly worse $\left(\delta_{z}=0.09\right.$ and $\left.c=12 \%\right)$.

Our photometry includes data from seven broad-band filters i.e. $V, I$ (UH8K Mosaic), $R$ (Keck/LRIS), and $z, J, H, K$ (Keck/NIRC). With respect to the HDF observations, we are missing data in the two bluest filters $(U$ and $B)$, but add in observations in the $R$ and $z$ bands. Bolzonella et al. (2000) determined that the lack of $U$-band photometry enhances the uncertainty only for objects at $z<0.2$. We expect a similar effect for the lack of $B$ data: enhanced uncertainties in the photometric redshifts primarily for lower redshift $(z<0.5)$ galaxies. Including accurate $z, J, H, K$ photometry strongly decreases the resulting dispersion within the $1<z<2$ range.

Here we consider the photometric redshifts for galaxies with $K<20$ and detected in more than 4 filters (37 objects). Fits to the eastern lobe used the full $V R I z J H K$ photometry. Fits to the western lobe used only $V R I H K$; no $z$ data were obtained, and the $J$ data had an unrecoverable zero point calibration due to nonphotometric conditions under which the data were obtained. The average errors at the $68 \%$ and $90 \%$ confidence levels are $\mathrm{d} z=0.05$ and $\mathrm{d} z=0.1$ in the eastern lobe and $\mathrm{d} z=0.1$ and $\mathrm{d} z=0.2$ in the western lobe. The larger uncertainties in the western lobe are due to the larger uncertainties in the near-infrared photometry and a more limited number of filters relative to the eastern lobe.

Both fields show a strong concentration of galaxies in the $z \sim 0.9-1.5$ range, as seen in Fig. 4. The mean redshift for the combined sample is $\left\langle z_{\mathrm{ph}}\right\rangle=1.26$ and the dispersion is $\sigma\left(z_{\mathrm{ph}}\right)=0.13$, consistent with our tests on the HDF-N. One of the objects at $z_{\mathrm{ph}}>2$ is the arc. In Fig. 3 we also show for comparison the redshift distribution expected in a pure luminosity evolution (PLE) model (Pozzetti et al. 1996) for $K<20$ field galaxies. We estimate a contamination from field galaxies of $\sim 21 \%$, i.e. $\sim 8$ galaxies. Since the median expected redshift of the field galaxies is $z_{\text {median }}=0.9$, we should see four foreground galaxies in our data, but only one is identified by our photometric redshift estimates. We note that some of the photometric redshift fits show secondary minima at low redshift. While these could be low redshift $(z<0.5)$ 


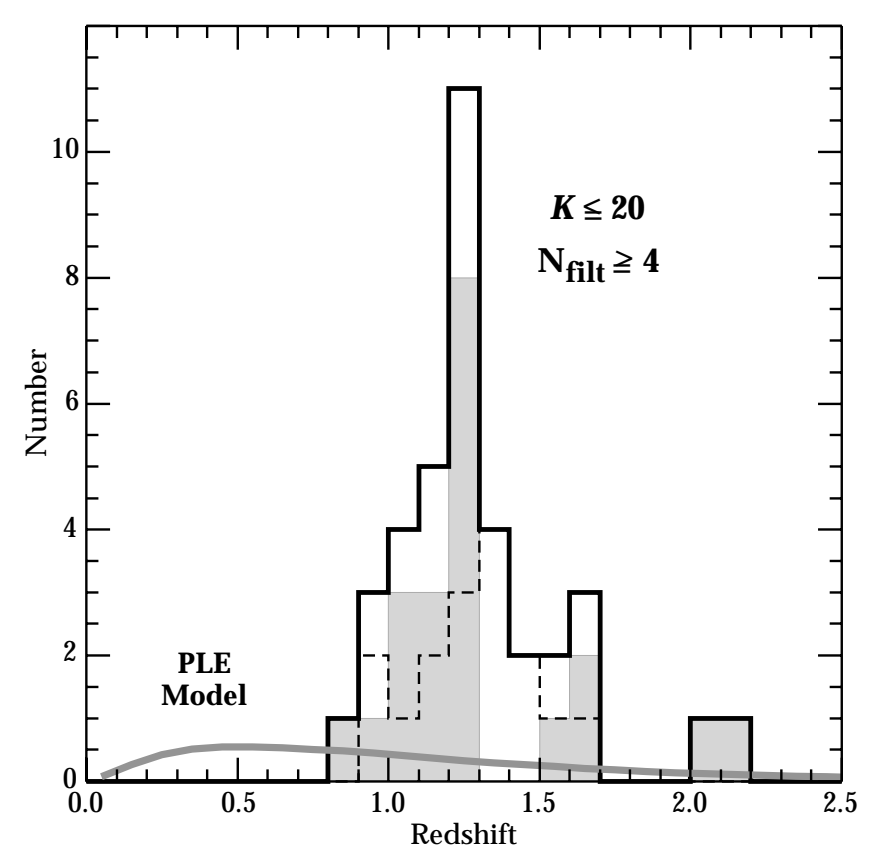

Fig. 4. A histogram of photometric redshifts for all galaxies with $K<20$ and detections in more than 4 filters in both of the $\mathrm{X}$-ray lobes. The gray histogram is the eastern lobe, while the western lobe is plotted with a dashed line. The combined data from both fields is plotted with a heavy black line. PLE model predictions are shown by the heavy gray line.

galaxies, we cannot break this degeneracy without the addition of $U$ and $B$ band data.

\subsection{The eastern lobe}

The presence of a gravitationally lensed giant arc in an extended X-ray source devoid of bright galaxies in the original Keck $R$-band image was sufficient to identify at least the eastern lobe of RX J105343+5735 as a distant cluster (Paper IV). We have used new photometry in the $V R I z J H K$ bands to place better constraints on the redshifts of the cluster galaxies, and obtained a spectroscopic redshift for the brightest cluster member. We also revise the cluster lensing mass (Paper IV) based on the new data.

The model fit to the photometry of the brightest galaxy in the eastern X-ray lobe of RX J105343+5735 is shown in Fig. 5. The galaxy is best fit with a Bruzual \& Charlot (1993) tau model with an e-folding time of 1 Gyr, a Salpeter IMF, solar metallicity, and an age of 6 Gyr at $z=1.27$. The fit assumes a low-density Universe $\left(\Omega_{\mathrm{m}}=0.1\right)$, where the age of the Universe at $z=1.27$ is $6.9 \mathrm{Gyr}$. This implies that the majority of the stars formed at much higher redshifts $(z>5)$ and the stellar population has undergone primarily passive evolution over the intervening time.

Using the photometric redshift estimate for the brightest galaxy in the eastern lobe, we obtained a near-infrared spectrum targeting the redshifted $\mathrm{H} \alpha$ line. The $H$-band spectrum obtained with NIRSPEC shows a strong emission line at $1.485 \pm 0.002 \mu \mathrm{m}$ (Fig. 6, upper panel). $\mathrm{H} \alpha$

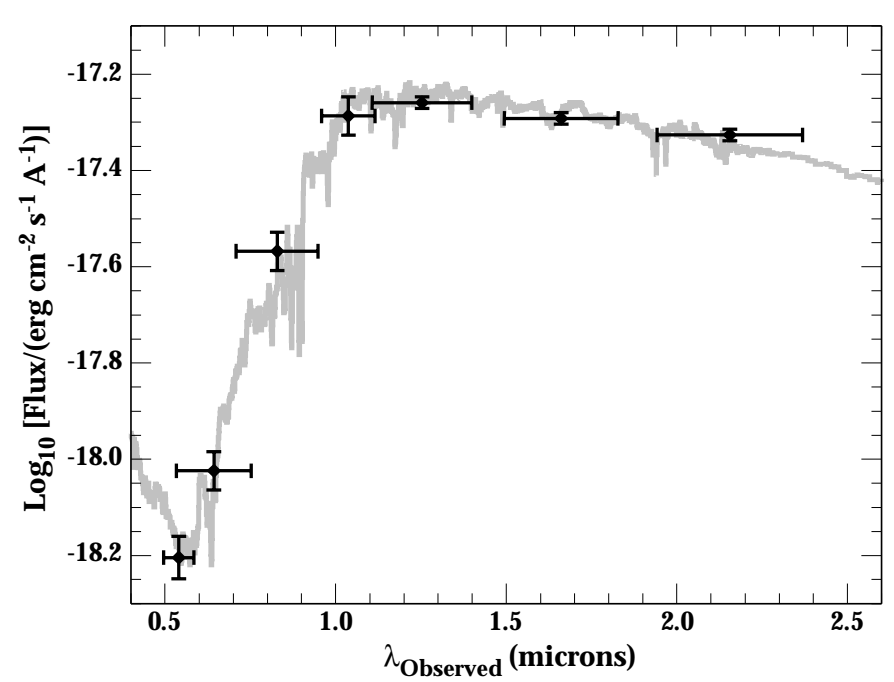

Fig. 5. The best-fit model spectrum (gray line) for the groundbased VRIzJHK photometry (points with error bars) of the brightest member of the eastern X-ray lobe. The photometric redshift is $z_{\text {phot }}=1.27$.

is the most reasonable interpretation for this line given the photometric redshift estimate. The observed equivalent width of the line is $\sim 100 \AA$, and there are few lines longward of $\mathrm{H} \alpha$ which could produce a line this strong. The only other possibility would be [O III] $\lambda \lambda 5007,4959 \AA$ or $\mathrm{H} \beta$, but we see only a single line. The emission line cannot have a rest wavelength shorter than $4150 \AA$, as this would place the cluster beyond the source at $z=$ 2.57 which is being lensed. We therefore interpret this as $\mathrm{H} \alpha$ emission, and the spectroscopic redshift is thus $1.263 \pm 0.003$.

The only difficulty with this interpretation is that the photometry is consistent with an old elliptical galaxy, which presumably would not have any strong ongoing star formation. Strong emission is not normally seen with cluster cooling flows. It is possible that the galaxy recently merged with a smaller, gas-rich companion, triggering a small starburst. However, without photometry in the $U$ and $B$ bands, which sample the restframe ultraviolet at this redshift, our photometric fits are not sensitive to the presence of some residual star formation.

Given the difficulties with the instrument when these data were obtained, we cannot report an emission line flux. The $\mathrm{H} \alpha$ line is, at best, marginally resolved at the instrumental resolution of $240 \mathrm{~km} \mathrm{~s}^{-1}$. However, strong nightsky $\mathrm{OH}$ lines bracketing the $\mathrm{H} \alpha$ line may affect this result. The line appears spatially extended, spanning $\sim 3^{\prime \prime} .5$. The line is also asymmetric about the center of the galaxy, extending $\sim 1^{\prime \prime}$. 0 east and $\sim 2$ ". 5 west.

The slit on NIRSPEC was oriented east-west, so the northern part of the lensed arc also fell on the slit. We found weak emission from [O II] $\lambda 3727 \AA$ at $1.333 \pm$ $0.004 \mu \mathrm{m}$ (Fig. 6, lower panel), yielding a redshift of $2.577 \pm 0.011$. The $[\mathrm{O}$ II] emission from the arc has a low signal-to-noise ratio and is affected by residuals from a strong night sky emission line which is on the blue side 


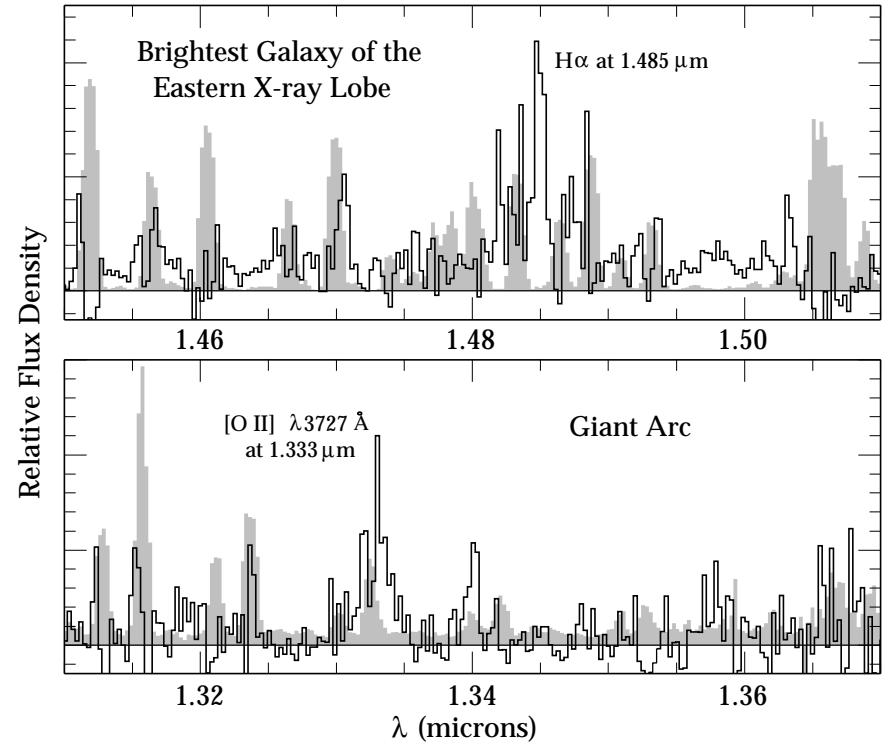

Fig. 6. NIRSPEC $H$-band spectra of the brightest member of the eastern X-ray lobe (upper panel), showing the $\mathrm{H} \alpha$ emission line redshifted to $1.485 \mu \mathrm{m}$, and the gravitationally lensed arc (lower panel), showing the [O II] $\lambda 3727 \AA$ line redshifted to $1.333 \mu \mathrm{m}$. In both panels, the background gray spectra show the locations and relative strengths of the night sky emission lines (scaled down by a factor of 800). While the $\mathrm{H} \alpha$ emission largely sits between $\mathrm{OH}$ lines, the [O II] line is affected by sky-subtraction residuals.

of the $[\mathrm{OII}]$ line center, so we refrain from making further conclusions about the lensed source. Our redshift is in good agreement with the $z=2.570$ determined in Paper IV from restframe ultraviolet metal absorption lines.

In Paper IV, Hasinger et al. determined a lensing mass of $0.4 \times 10^{14} M_{\odot}$ in the eastern lobe based on the curvature of the lensed arc in the Keck $R$-band data and an assumed redshift $(z=1.2)$ for the lensing cluster. We set more accurate constraints using the higher resolution $K$-band image and spectroscopic redshifts.

Following the derivation of Narayan \& Bartelmann (1999), we assume that the cluster potential can be approximated as a singular isothermal sphere, and that the radius of curvature for the lensed arc corresponds to the Einstein radius for the enclosed mass. The mean surface mass density is assumed to be the critical density $\left(\Sigma_{\text {crit }}\right)$ for strong lensing. The mass within the arc is then simply this mean density multiplied by the crosssectional area within the arc: $M(\leq \theta)=\pi \Sigma_{\text {crit }}\left(D_{\mathrm{d}} \theta\right)^{2}$, where $\theta$ is the radius of curvature of the arc, and $D_{\mathrm{d}}$ is the angular diameter distance to the lensing cluster (the deflector). The critical surface density term contains a factor which is dependent on the lensing geometry. From the $K$-band image we estimate the radius of curvature for the arc to be $4 . \prime 7 \pm 1^{\prime \prime} .0$, corresponding to $40 \mathrm{kpc}$ at $z=1.263$. With $z_{\text {lens }}=1.263$ and $z_{\text {arc }}=2.577$ the lensing mass is $1.7 \pm 0.7 \times 10^{13} M_{\odot}$. As noted in Paper IV, this corresponds only to the central region of one of the two lobes of RX J105343+5735.

We note that the center of curvature of the arc does not coincide with the position of the brightest galaxy in the eastern lobe, but lies $\sim 2^{\prime \prime}$ to the northwest, in the direction of the second brightest galaxy (see Fig. 2), perhaps indicating that the brightest galaxy in the eastern lobe does not lie at the dynamical center of the cluster.

\subsection{The western lobe}

Without spectroscopic redshifts for any of the galaxies in the western lobe, our discussion here is necessarily somewhat speculative. We base our conclusions about the western X-ray lobe primarily on three observations. First, chance alignment of two unrelated clusters is extremely unlikely. Second, the two lobes contain galaxies with similar colors. Third, alignment in the morphology of the $\mathrm{X}$-ray lobes suggests interaction.

Perhaps the strongest argument in favor of association is the relative rarity of clusters at high redshift. Rosati et al. (2000) find a space density of $z \sim 1$ clusters in the ROSAT Deep Cluster Survey of $\sim 3 \times 10^{-7} \mathrm{Mpc}^{-3}$. The corresponding surface density over the redshift range of $1.0-1.5$ would be only $\sim 2.2$ clusters per square degree. The probability of X-ray detection of two unrelated highredshift clusters separated by $\sim 1$ arcmin, corresponding to a projected separation of $0.5 \mathrm{Mpc}$, in the $\sim 0.2$ square degrees covered by the HRI Ultra Deep Survey is quite low.

Another argument in favor of association is the similarity of colors for galaxies in both lobes. Figure 3 shows the $(R-K)$ and $(I-K)$ vs. $K$ color-magnitude diagrams. In particular, the brightest several galaxies in each lobe form a single, strong color sequence fully consistent with and photometrically indistinguishable from galaxies in other, known clusters at the same redshift. The photometric redshifts (Fig. 4) also suggest similar redshifts for the two lobes. The broader distribution of redshifts in the western lobe is mostly attributable to the smaller number of filters for which we have reliable photometry.

Finally, both of the X-ray lobes in RX J105343+5735 show an elongated morphology which is aligned along the direction of the separation between the two X-ray peaks (Fig. 1), suggesting gravitational interaction. We would expect a random orientation in the relative morphology of two unrelated clusters.

\section{Conclusions}

We have confirmed that the eastern X-ray lobe of RX J105343+5735 is a massive cluster of galaxies at a redshift of $z=1.263 \pm 0.003$ on the basis of a single strong emission line, interpreted as $\mathrm{H} \alpha$, in the brightest galaxy in this lobe. The lensing mass is estimated to be $1.7 \pm 0.4 \times 10^{13} M_{\odot}$. This is the mass within the $4{ }^{\prime \prime} 7 \mathrm{ra}-$ dius of curvature of the arc, which corresponds only to the central $\sim 40 \mathrm{kpc}$ radius centered on the eastern lobe. 
The total cluster mass will be larger. Detection of [O II] $\lambda 3727 \AA$ emission at $z=2.577 \pm 0.011$ from the lensed arc confirms a prior measurement.

Based on the similarities in color between galaxies in the two lobes, the low probability of finding two unrelated $\mathrm{X}$-ray clusters in the HRI data separated by $\sim 1$ arcmin $(0.5 \mathrm{Mpc})$, and an aligned morphology in the X-ray image, we conclude that the western lobe is most likely an associated cluster at the same redshift. The system may thus represent a pair of galaxy clusters or subclumps in the process of merging. Recent observations of this cluster with XMM (Hashimoto et al. 2001) come to a similar conclusion. The system as a whole represents a moderately large cluster of galaxies.

Acknowledgements. DT and BTS acknowledge support from NSF and NASA grants. GH and GS acknowledge support from the DLR grant 50 OR 9908 0. LP and GZ acknowledge support provided by ASI through contracts ASI-ARS-99-15 and ASII/R/35/00. Data presented herein were obtained at the W.M. Keck Observatory, which is operated as a scientific partnership among the California Institute of Technology, the University of California and the National Aeronautics and Space Administration. The Observatory was made possible by the generous financial support of the W.M. Keck Foundation.

\section{References}

Avni, Y. 1976, ApJ, 210, 642

Benítez, N., Broadhurst, T., Rosati, P., et al. 1999, ApJ, 527, 31

Bolzonella, M., Miralles, J.-M., \& Pello, R. 2000, A\&A, 363, 476

Bruzual, A. C., \& Charlot, S. 1993, ApJ, 405, 538

Carilli, C. L., Harris, D. E., Pentericci, L., et al. 1998, ApJ, 494, L143

Connolly, A. J., Szalay, A. S., Dickinson, M., Subbarao, M. U., \& Brunner, R. J. 1997, ApJ, 486, L11
Daddi, E., Cimatti, A., Pozzetti, L., et al. 2000, A\&A, 361, 535

Fernandez-Soto, A., Lanzetta, K. M., \& Yahil, A. 1999, ApJ, 513, 34

Hall, P. B., Green, R. F., \& Cohen, M. 1998, ApJSS, 119, 1

Hashimoto, Y., Hasinger, G., Arnaud, M., Rosati, P., \& Miyaji, T. 2001, A\&A, submitted

Hasinger, G., Giacconi, R., Gunn, J. E., et al. 1998, A\&A, 340, L27 (Paper IV)

Landolt, A. U. 1992, AJ, 104, 340

Lehmann, I., Hasinger, G., Schmidt, M., et al. 2001, A\&A, 371, 833 (Paper VI)

Lowenthal, J. D., Koo, D. C., Guzman, R., et al. 1997, ApJ, 481,673

Madau, P. 1995, ApJ, 441, 18

Matthews, K., \& Soifer, B. T. 1994, in Infrared Astronomy with Arrays: the Next Generation, ed. I. McLean (Dordrecht: Kluwer Academic Publishers), 239

McLean, I. S., Becklin, E. E., Bendiksen, O., et al. 1998, in Infrared Astronomical Instrumentation, ed. A. M. Fowler, Proc. SPIE, 3354, 566

Narayan, R., \& Bartelmann, M. 1999, Chapter 10 in Formation of Structure in the Universe, ed. A. Dekel, \& J. Ostriker (Cambridge University Press), 360

Persson, S. E., Murphy, D. E., Krzeminski, W., Roth, M., \& Rieke, M. J. 1998, AJ, 116, 2745

Pozzetti, L., Bruzual, A., Zamorani, G. 1996, MNRAS, 281, 953

Rosati, P., Stanford, S. A., Eisenhardt, P. R., et al. 1999, AJ, 118,76

Rosati, P., Lidman, C., Della Ceca, R., et al. 2000, ESO Messenger, 99, 26

Stanford, S. A., Elston, R., Eisenhardt, P. R., et al. 1997, AJ, 114, 2232

Steidel, C. C., Giavalisco, M., Dickinson, M., \& Adelberger, K. L. 1996, AJ, 112, 352

Williams, R. E., Blacker, B., Dickinson, M., et al. 1996, AJ, 112,1335

Wilson, G., \& Kaiser, N. 2001, PASP, in preparation 